\title{
Non-linear sigma models via the chiral de Rham complex
}

\author{
Joel Ekstrand ${ }^{1}$, Reimundo Heluani ${ }^{2}$, Johan Källén ${ }^{1}$ \\ and Maxim Zabzine ${ }^{1}$ \\ ${ }^{1}$ Department of Physics and Astronomy, Uppsala University, \\ Box 516, SE-75120 Uppsala, Sweden \\ maxim.zabzine@fysast.uu.se \\ ${ }^{2}$ Department of Mathematics, University of California, \\ Berkeley, CA 94720, USA
}

\begin{abstract}
We propose a physical interpretation of the chiral de Rham complex as a formal Hamiltonian quantization of the supersymmetric non-linear sigma model. We show that the chiral de Rham complex on a Calabi-Yau manifold carries all information about the classical dynamics of the sigma model. Physically, this provides an operator realization of the non-linear sigma model. Mathematically, the idea suggests the use of Hamiltonian flow equations within the vertex algebra formalism with the possibility to incorporate both left and right moving sectors within one mathematical framework.
\end{abstract}

\section{Introduction}

The chiral de Rham complex (CDR) is a notion introduced by the mathematicians Malikov et al. [16]. CDR is a sheaf of supersymmetric vertex

e-print archive: http://lanl.arXiv.org/abs/0612164 
algebras over a smooth manifold $M$. It is defined by gluing free chiral algebras on the overlaps of open subsets of $M$ isomorphic to $\mathbb{R}^{n}\left(\mathbb{C}^{n}\right)$. Since the original article [16], there has been considerable progress on the mathematical literature about CDR. In physics, however, CDR did not attract extensive attention. The keyword in the physics interpretation of CDR were the words "chiral" and "perturbative". In [14,19-21] CDR (and more generally, chiral differential operators) were interpreted in the context of the half-twisted sigma model in the perturbative regime. In [17] a Lagrangian approach to CDR and other related sheaves was described. In another set of related works, [7-9], CDR was discussed in the context of the infinite volume limit of the sigma model. The infinite volume sigma model was suggested as a "non-perturbative completion of CDR".

In this work, we would like to initiate a different interpretation of CDR. Our idea originates from the observation that many formulas in two seemingly unrelated subjects are identical. Namely, the formulas originating from the study of CDR $[2,11,12]$ are identical (modulo some quantum terms) to the formulas arising within the classical Hamiltonian analysis of the $N=(1,1)$ supersymmetric non-linear sigma model $[4,22,23]$. Moreover, the Poisson brackets in the Hamiltonian formalism agree up to quantum terms with the quantum brackets in CDR. This fact strongly suggests to interpret CDR as a formal canonical quantization of the non-linear sigma model. However, CDR is just a formal quantization of the string phase space, and it does not carry any dynamical information unless the Hamiltonian flow equations are introduced in the game. If this is done, then CDR, with a particular choice of a global section, encodes everything about the classical dynamics of the non-linear sigma model. Hopefully, it knows about the quantum dynamics as well.

One peculiarity of the Hamiltonian formalism is that it mixes the left (chiral) and right (anti-chiral) moving sectors. Thus, taking seriously the Hamiltonian interpretation of CDR, the word "chiral" in CDR becomes misleading. If properly interpreted, CDR carries information about both the chiral and the anti-chiral sector of theory. ${ }^{1}$ Mathematically, it gives hope to recover the chiral and anti-chiral sectors within the framework of vertex algebras and therefore it may provide, eventually, a more solid mathematical basis for the study of non-linear sigma models.

The paper is organized as follows. In Section 2 we briefly review the formalism of vertex algebras and the definition of CDR as a family of sheaves of SUSY vertex algebras. In Section 3 we discuss a particular set of global sections of CDR on a Calabi-Yau manifold which gives rise to two commuting

\footnotetext{
${ }^{1}$ The name chiral-anti-chiral de Rham complex was suggested in [9].
} 
copies of the $N=2$ superconformal algebra. Section 4 reviews some basics about the classical $N=(1,1)$ supersymmetric sigma models and its Hamiltonian treatment. We recover the above-mentioned global sections of CDR within the Hamiltonian formalism. We also discuss the classical equations of motion. Section 5 contains an interpretation of CDR as a formal canonical quantization of the non-linear sigma model. We also show in this section how CDR suggests the way to calculate equal time commutators for the sigma model. We also briefly discuss the mathematical aspects of the present interpretation. In Section 6 we present rather simple examples of interpreting the free boson and free fermion in terms of CDR. Section 7 contains final remarks and further speculations on the present interpretation of CDR.

Before proceeding further, let us make a disclaimer. This is a physics article, not a mathematical one. However, we believe that the present physical ideas bring along interesting mathematics and thus we allow ourselves for some short speculations on this subject. We hope to return to proper mathematical treatment of these ideas in a separate publication.

\section{Review of CDR}

In this section we review the basics of vertex algebras and CDR. We also set the conventions for the rest of the article. We use the name "chiral de Rham complex" (CDR) due to historical reasons. Otherwise, we find the name CDR misleading, especially in the context of the present work. To avoid any fixation on the word "chiral" we denote our formal coordinate for the punctured disk by $\xi$. In later discussions, we associate $\xi=\mathrm{e}^{i \sigma}$ with the periodic coordinate $\sigma$ along the loop. CDR was originally introduced in [16], however, we follow the treatment given in [2].

First, let us review the definition of vertex superalgebras, as presented in [13]. Given a vector space $V$, an $\operatorname{End}(\mathrm{V})$-valued field is a formal distribution of the form

$$
A(\xi)=\sum_{n \in \mathbb{Z}} \xi^{-1-n} A_{(n)}, \quad A_{(n)} \in \operatorname{End}(\mathrm{V}),
$$

such that for every $v \in V$, we have $A_{(n)} v=0$ for large enough $n$.

A vertex superalgebra consists of the data of a supervector space $V$, an even vector $|0\rangle \in V$ (the vacuum vector), an even endomorphism $\partial$, and a parity preserving linear map $A \mapsto Y(A, \xi)$ from $V$ to $\operatorname{End}(\mathrm{V})$-valued fields (the state-field correspondence). This data should satisfy the following set of axioms: 
- Vacuum axioms:

$$
\begin{aligned}
Y(|0\rangle, \xi) & =\mathrm{Id}, \\
Y(A, \xi)|0\rangle & =A+O(\xi), \\
\partial|0\rangle & =0 .
\end{aligned}
$$

- Translation invariance: ${ }^{2}$

$$
[\partial, Y(A, \xi)]=\partial_{\xi} Y(A, \xi) .
$$

- Locality:

$$
\left(\xi-\xi^{\prime}\right)^{n}\left[Y(A, \xi), Y\left(B, \xi^{\prime}\right)\right]=0, \quad n \gg 0 .
$$

(The notation $O(\xi)$ denotes a power series in $\xi$ without constant term.)

Given a vertex superalgebra $V$ and a vector $A \in V$, we expand the fields

$$
Y(A, \xi)=A(\xi)=\sum_{j \in \mathbb{Z}} \xi^{-1-j} A_{(j)},
$$

and we call the endomorphisms $A_{(j)}$ the Fourier modes of $Y(A, \xi)$. Now, define the operations

$$
\begin{aligned}
& {\left[A_{\lambda} B\right]=\sum_{j \geq 0} \frac{\lambda^{j}}{j !} A_{(j)} B,} \\
& : A B:=A_{(-1)} B,
\end{aligned}
$$

where $\lambda$ is a formal even parameter. The first operation is called the $\lambda$-bracket and the second is called the normally ordered product. The $\lambda$-bracket contains all the information about the commutators between the Fourier coefficients of fields in $V$, and the OPE can easily be read from it, namely

$$
A(\xi) B\left(\xi^{\prime}\right)=\sum_{j \geq 0} \frac{\left(A_{(j)} B\right)\left(\xi^{\prime}\right)}{\left(\xi-\xi^{\prime}\right)^{j+1}}+: A(\xi) B\left(\xi^{\prime}\right):
$$

where the last term corresponds to the normally ordered product. Correspondingly, the commutator is

$$
\left[A(\xi), B\left(\xi^{\prime}\right)\right]=\sum_{j \geq 0} \frac{1}{j !}\left(A_{(j)} B\right)\left(\xi^{\prime}\right) \partial_{\xi^{\prime}}^{j} \delta\left(\xi-\xi^{\prime}\right) .
$$

\footnotetext{
${ }^{2}$ We denote the even endomorphism and the derivative along $\xi$ by the same sign $\partial$. The appropriate interpretation of $\partial$ should be clear from the context.
} 
In all further considerations we drop the notation : :, and the normal ordering is always assumed in the quantum setup. In practice, the $\lambda$-bracket is a convenient tool for manipulations with OPEs.

Next, we review SUSY vertex algebras from [10]. The idea behind the definition is to extend the formal even variable $\xi$ to formal variables $(\xi, \theta)$ with $\theta$ being an odd Grassman variable. Given a super vector space $V$, an End $(\mathrm{V})$-valued superfield is a formal distribution of the form

$$
A(\xi, \theta)=\sum_{\substack{j \in \mathbb{Z} \\ J=0,1}} \xi^{-1-j} \theta^{1-J} A_{(j \mid J)}, \quad A_{(j \mid J)} \in \operatorname{End}(\mathrm{V})
$$

such that for every $v \in V, A_{(j \mid J)} v=0$ for large enough $j$.

A SUSY vertex algebra consists of the data of a supervector space $V$, an even vector $|0\rangle \in V$ (the vacuum vector), an odd endomorphism $D$ (whose square is an even endomorphism which we denote $\partial$ ), and a parity preserving linear map $A \mapsto Y(A, \xi, \theta)$ from $V$ to $\operatorname{End}(\mathrm{V})$-valued superfields (the statesuperfield correspondence). This data should satisfy the following set of axioms:

- Vacuum axioms:

$$
\begin{aligned}
Y(|0\rangle, \xi, \theta) & =\mathrm{Id}, \\
Y(A, \xi, \theta)|0\rangle & =A+O(\xi, \theta), \\
D|0\rangle & =0 .
\end{aligned}
$$

- Translation invariance:

$$
\begin{aligned}
{[D, Y(A, \xi, \theta)] } & =\left(\partial_{\theta}-\theta \partial_{\xi}\right) Y(A, \xi, \theta), \\
{[\partial, Y(A, \xi, \theta)] } & =\partial_{\xi} Y(A, \xi, \theta) .
\end{aligned}
$$

- Locality:

$$
\left(\xi-\xi^{\prime}\right)^{n}\left[Y(A, \xi, \theta), Y\left(B, \xi^{\prime}, \theta^{\prime}\right)\right]=0, \quad n \gg 0 .
$$

(The notation $O(\xi, \theta)$ denotes a power series in $\xi$ and $\theta$ without a constant term in $\xi$.)

Given the vacuum axioms for a SUSY vertex algebra, we will use the state-field correspondence to identify a vector $A \in V$ with its corresponding field $Y(A, \xi, \theta)$. Given a SUSY vertex algebra $V$ and a vector $A \in V$, we 
expand the fields

$$
Y(A, \xi, \theta)=A(\xi, \theta)=\sum_{\substack{j \in \mathbb{Z} \\ J=0,1}} \xi^{-1-j} \theta^{1-J} A_{(j \mid J)}
$$

and we call the endomorphisms $A_{(j \mid J)}$ the Fourier modes of $Y(A, \xi, \theta)$. Define now the operations

$$
\begin{aligned}
{\left[A_{\Lambda} B\right] } & =\sum_{\substack{j \geq 0 \\
J=0,1}} \frac{\Lambda^{j \mid J}}{j !} A_{(j \mid J)} B \\
A B & =A_{(-1 \mid 1)} B
\end{aligned}
$$

where $\Lambda^{j \mid J}=\lambda^{j} \chi^{J}$, with formal even $\lambda$ and odd $\chi$ satisfying $\chi^{2}=-\lambda$. The first operation is called the $\Lambda$-bracket and the second is called the normally ordered product. This $\Lambda$-bracket is an efficient way to encode and manipulate OPEs of superfields. In particular, the commutator of two superfields is given by the following expression:

$$
\left[A(\xi, \theta), B\left(\xi^{\prime}, \theta^{\prime}\right)\right]=\sum_{\substack{j \geq 0 \\ J=0,1}} \frac{(-1)^{J}}{j !}\left(\partial_{\xi^{\prime}}^{j} D_{\xi^{\prime} \theta^{\prime}}^{J} \delta\left(\xi-\xi^{\prime}\right) \delta\left(\theta-\theta^{\prime}\right)\right)\left(A_{(j \mid J)} B\right)\left(\xi^{\prime}, \theta^{\prime}\right) .
$$

As in the standard setting, given a SUSY vertex algebra $V$ and a vector $A \in V$, we have:

$$
Y(\partial A, \xi, \theta)=\partial_{\xi} Y(A, \xi, \theta)=[\partial, Y(A, \xi, \theta)]
$$

On the other hand, the action of the derivation $D$ is described by

$$
Y(D A, \xi, \theta)=\left(\partial_{\theta}+\theta \partial_{\xi}\right) Y(A, \xi, \theta) \neq[D, Y(A, \xi, \theta)]
$$

For further details of the formalism the reader may consult [10].

In [16], given any smooth manifold $M$, the authors introduced a sheaf of vertex algebras on $M$ which they called the CDR. Roughly, the idea is to associate locally over a neighborhood of a point, a vertex algebra corresponding to a free $\beta \gamma b c$-system. The crucial observation is that the group of coordinate changes can be mapped into the group of vertex algebra automorphisms of this free system. This allows one to glue the algebras associated to different open sets in $M$ together, and construct a sheaf. Although the formalism of [16] works in the analytic, algebraic and smooth settings, most of the mathematics literature on CDR is dedicated to the algebraic case. In 
the present case, we are interested in the smooth setting. This set-up was considered in [15] and, in the superfield formulation, in [2].

The $\beta \gamma$-system is the vertex algebra generated by the even fields $\beta_{\mu}$ and $\gamma^{\mu}$, subject to the following relation:

$$
\left[\beta_{\mu}(\xi), \gamma^{\nu}\left(\xi^{\prime}\right)\right]=\hbar \delta_{\mu}^{\nu} \delta\left(\xi-\xi^{\prime}\right)
$$

The fermionic $b c$-system is the vertex algebra generated by the odd fields $b_{\mu}$ and $c^{\mu}$ satisfying

$$
\left[c^{\mu}(\xi), b_{\nu}\left(\xi^{\prime}\right)\right]_{+}=\hbar \delta_{\nu}^{\mu} \delta\left(\xi-\xi^{\prime}\right),
$$

where $[,]_{+}$stands for anticommutator. In the following discussion, all our operations will be $\mathbb{Z}_{2}$-graded, and we will drop the subscript + . In Section 6 we will consider the $\beta \gamma b c$-system in more detail. We can combine these fields into superfields and introduce the SUSY vertex algebra generated by

$$
\phi^{\mu}(\xi, \theta)=\gamma^{\mu}(\xi)+\theta c^{\mu}(\xi), \quad S_{\mu}(\xi, \theta)=b_{\mu}(\xi)+\theta \beta_{\mu}(\xi),
$$

which satisfy

$$
\left[\phi^{\mu}(\xi, \theta), S_{\nu}\left(\xi^{\prime}, \theta^{\prime}\right)\right]=\hbar \delta_{\nu}^{\mu} \delta\left(\xi-\xi^{\prime}\right) \delta\left(\theta-\theta^{\prime}\right),
$$

or equivalently

$$
\left[\phi^{\mu}{ }_{\Lambda} S_{\nu}\right]=\hbar \delta_{\nu}^{\mu}
$$

From (2.8) we easily recover the collection of the standard $\beta \gamma$-system (2.5) and $b c$-system (2.6). Given a change of coordinates $\tilde{x}^{\mu}=g^{\mu}(x)$ with its inverse $x^{\mu}=f^{\mu}(\tilde{x})$, we can define a SUSY vertex algebra automorphism as follows:

$$
\tilde{\phi}^{\mu}=g^{\mu}(\phi), \quad \tilde{S}_{\mu}=\left(\frac{\partial f^{\mu}}{\partial \tilde{\phi}^{\nu}}(g(\phi)) S_{\nu}\right) .
$$

Recall that products are normally ordered. The automorphism (2.9) preserves, in particular, relation (2.8). Using (2.9) we can glue the SUSY vertex algebras generated by (2.8) to obtain a sheaf $\Omega_{\hbar}^{\text {ch }}(M)$ of SUSY vertex algebras over $M$. Indeed, we deal with a family of sheaves which depends (polynomially) on $\hbar$. It is important to stress that at this moment we do not need to discuss any allocation of conformal weights for the fields $\phi^{\mu}$ and $S_{\mu}$. The set of global sections $\Gamma\left(M, \Omega_{\hbar}^{\mathrm{ch}}(M)\right)$ give rise to a family (depending on $\hbar)$ of SUSY vertex algebras attached to $M$. 


\section{CDR on Calabi-Yau manifolds}

In this section, we construct a specific collection of global sections of $\Omega_{\hbar}^{\text {ch }}(M)$ on any Calabi-Yau manifold. In our presentation, we borrow the results from $[2,11]$.

On any orientable manifold $M$, with the choice of volume form

$$
\operatorname{vol}_{d}=\mathrm{e}^{\rho(x)} d x^{1} \wedge \cdots \wedge d x^{d}
$$

the following defines a global section of $\Omega_{\hbar}^{\text {ch }}(M)$ :

$$
\mathcal{P}=D \phi^{\mu} D S_{\mu}+\partial \phi^{\mu} S_{\mu}-\hbar \partial D \rho
$$

Expanded as

$$
\mathcal{P}(\xi, \theta)=G(\xi)+2 \theta L(\xi),
$$

it generates the $N=1$ superconformal algebra $(G, L)$ with central charge $3 \operatorname{dim} M$. With respect to this Virasoro generator $L$, the fields $\phi^{\mu}$ are primary of conformal weight 0 . The fields $S_{\mu}$ have conformal weight $1 / 2$, but are not primary unless we choose coordinates where the volume form is constant. We stress that this assignment of conformal weights is not the one that usually is considered in the literature, in particular, it differs from the one in the original work [16]. For further details, see Section 6.5 below.

Let $M$ be a complex manifold with complex structure $I$, and suppose it admits a closed holomorphic volume form $\Omega$ :

$$
\Omega=\mathrm{e}^{f(z)} d z^{1} \wedge \cdots \wedge d z^{n}
$$

written in holomorphic coordinates, with $\operatorname{dim}_{\mathbb{R}} M=d=2 n$. $\Omega$ is related to the real volume form by

$$
\Omega \wedge \bar{\Omega}=\mathrm{i}^{n(n+2)} 2^{n} \operatorname{vol}_{d} .
$$

In this case, $\Omega_{\hbar}^{\text {ch }}(M)$ has the following global section:

$$
\mathcal{J}_{1}=\left(I_{\nu}^{\mu} D \phi^{\nu}\right) S_{\mu}+\frac{i}{2} \hbar \partial(f-\bar{f})
$$

such that

$$
\begin{aligned}
\mathcal{J}_{1}(\xi, \theta) & =-\mathrm{i} J_{1}(\xi)-\mathrm{i} \theta\left(G_{1}^{-}(\xi)-G_{1}^{+}(\xi)\right), \\
\mathcal{P}(\xi, \theta) & =\left(G_{1}^{+}(\xi)+G_{1}^{-}(\xi)\right)+2 \theta L_{1}(\xi),
\end{aligned}
$$


and $\left(J_{1}, G_{1}^{ \pm}, L_{1}\right)$ generate the $N=2$ superconformal algebra of central charge $3 \operatorname{dim} M$.

If the manifold is symplectic with the symplectic form $\omega$, then CDR admits the following global section:

$$
\mathcal{J}_{2}=\frac{1}{2}\left(\omega^{\mu \nu} S_{\mu} S_{\nu}-\omega_{\mu \nu} D \phi^{\mu} D \phi^{\nu}\right),
$$

with $\omega_{\mu \nu} \omega^{\nu \lambda}=\delta_{\mu}^{\lambda}$, such that

$$
\begin{gathered}
\mathcal{J}_{2}(\xi, \theta)=-\mathrm{i} J_{2}(\xi)-\mathrm{i} \theta\left(G_{2}^{-}(\xi)-G_{2}^{+}(\xi)\right), \\
\mathcal{P}(\xi, \theta)=\left(G_{2}^{+}(\xi)+G_{2}^{-}(\xi)\right)+2 \theta L_{2}(\xi),
\end{gathered}
$$

and $\left(J_{2}, G_{2}^{ \pm}, L_{2}\right)$ generate the $N=2$ superconformal algebra of central charge $3 \operatorname{dim} M$. The existence of the global sections (3.2) and (3.5) and their relation to $N=2$ superconformal algebra is a generic feature of generalized Calabi-Yau manifolds [12].

Let $M$ be a Calabi-Yau manifold and choose a Ricci flat metric $g$, a complex structure $I$ and a closed Kähler form $\omega=g I$. In this case $\rho=$ $\log \sqrt{\operatorname{det}\left(g_{\mu \nu}\right)}$ and, in addition to the sections (3.1), (3.2) and (3.5), one can define another global section ${ }^{3}$

$$
\mathcal{H}=\partial \phi^{\mu} D \phi^{\nu} g_{\mu \nu}+g^{\mu \nu} D S_{\mu} S_{\nu}+\Gamma^{\rho}{ }_{\sigma \nu} g^{\nu \lambda} D \phi^{\sigma}\left(S_{\lambda} S_{\rho}\right),
$$

where $\Gamma$ is the Levi-Civita connection. The algebraic relations satisfied by these global sections $\left(\mathcal{P}, \mathcal{J}_{1}, \mathcal{J}_{2}, \mathcal{H}\right)$ are given in the appendix, both in terms of $\Lambda$-brackets: (A.1) to (A.10), and of their commutators: (A.11) to (A.18). Furthermore, we define the following global sections:

$$
\begin{aligned}
\mathcal{H}_{\mathrm{L}} & =\frac{1}{2}(\mathcal{P}+\mathcal{H}), \quad \mathcal{H}_{\mathrm{R}}=\frac{1}{2}(\mathcal{P}-\mathcal{H}), \quad \mathcal{J}_{\mathrm{L}}=\frac{1}{2}\left(\mathcal{J}_{1}+\mathcal{J}_{2}\right), \\
\mathcal{J}_{\mathrm{R}} & =\frac{1}{2}\left(\mathcal{J}_{1}-\mathcal{J}_{2}\right),
\end{aligned}
$$

such that

$$
\begin{aligned}
& \mathcal{J}_{\mathrm{L}}(\xi, \theta)=-\mathrm{i} J_{\mathrm{L}}(\xi)-\mathrm{i} \theta\left(G_{\mathrm{L}}^{-}(\xi)-G_{\mathrm{L}}^{+}(\xi)\right), \\
& \mathcal{H}_{\mathrm{L}}(\xi, \theta)=\left(G_{\mathrm{L}}^{+}(\xi)+G_{\mathrm{L}}^{-}(\xi)\right)+2 \theta L_{\mathrm{L}}(\xi), \\
& \mathcal{J}_{\mathrm{R}}(\xi, \theta)=-\mathrm{i} J_{\mathrm{R}}(\xi)-\mathrm{i} \theta\left(G_{\mathrm{R}}^{-}(\xi)-G_{\mathrm{R}}^{+}(\xi)\right), \\
& \mathcal{H}_{\mathrm{R}}(\xi, \theta)=\left(G_{\mathrm{R}}^{+}(\xi)+G_{\mathrm{R}}^{-}(\xi)\right)+2 \theta L_{\mathrm{R}}(\xi) .
\end{aligned}
$$

\footnotetext{
${ }^{3} \mathcal{H}$ is a global section on a Calabi-Yau manifold, see [11] for further details. It is not clear whether or not it is a global section on a generic Riemannian manifold.
} 
A tedious calculation from [11] shows that $\left(J_{\mathrm{L}}, G_{\mathrm{L}}^{ \pm}, L_{\mathrm{L}}\right)$ and $\left(J_{\mathrm{R}}, G_{\mathrm{R}}^{ \pm}, L_{\mathrm{R}}\right)$ generate two commuting copies of the $N=2$ superconformal algebra with central charge $\frac{3}{2} \operatorname{dim} M$ each. The relation between the different $N=2$ algebras is given by the following expressions:

$$
\begin{aligned}
L & =L_{1}=L_{2}=L_{\mathrm{L}}+L_{\mathrm{R}} \\
J_{1} & =J_{\mathrm{L}}+J_{\mathrm{R}}, \quad J_{2}=J_{\mathrm{L}}-J_{\mathrm{R}}, \\
G_{1}^{ \pm} & =G_{\mathrm{L}}^{ \pm}+G_{\mathrm{R}}^{ \pm}, \quad G_{2}^{ \pm}=G_{\mathrm{L}}^{ \pm}+G_{\mathrm{R}}^{\mp} \\
G & =G_{1}^{+}+G_{1}^{-}=G_{2}^{+}+G_{2}^{-} .
\end{aligned}
$$

Summarizing, CDR enables us to construct four different $N=2$ superconformal algebras on a Calabi-Yau manifold with fixed complex and Kähler moduli. There are two commuting copies $\left(J_{\mathrm{L}}, G_{\mathrm{L}}^{ \pm}, L_{\mathrm{L}}\right)$ and $\left(J_{\mathrm{R}}, G_{\mathrm{R}}^{ \pm}, L_{\mathrm{R}}\right)$, with central charge $\frac{3}{2} \operatorname{dim} M$ each. Their different "diagonal" combinations (3.14) to (3.17) give rise to the $N=2$ algebra $\left(J_{1}, G_{1}^{ \pm}, L_{1}\right)$ which depends only on the complex moduli; and to the $N=2$ algebra $\left(J_{2}, G_{2}^{ \pm}, L_{2}\right)$ which depends only on the Kähler moduli.

The following is a simple but important observation about these two commuting copies of the $N=2$ superconformal algebra. Let us map the variable $\xi$ to a loop coordinate $\sigma$ such that $\xi=\mathrm{e}^{\mathrm{i} \sigma}$. The transformations will be controlled by the diagonal Virasoro algebra $L$, see (3.14). Thus a field $O(\xi)$, primary of conformal weight $\Delta$ :

$$
O(\xi)=\sum_{n \in-\Delta+\mathbb{Z}} O_{n} \xi^{-n-\Delta},
$$

has the following expansion with respect to $\sigma$ :

$$
O(\sigma)=\mathrm{i}^{\Delta} \sum_{n \in \mathbb{Z}} O_{n} \mathrm{e}^{-\mathrm{i} n \sigma} .
$$

We may regard these operators as operators at time zero within the canonical quantization on a cylinder $S^{1} \times \mathbb{R}$. We choose the Hamiltonian

$$
H=\mathrm{i}\left(L_{\mathrm{L}}\right)_{0}-\mathrm{i}\left(L_{\mathrm{R}}\right)_{0}=\frac{\mathrm{i}}{2} \int d \xi d \theta \xi \mathcal{H}(\xi, \theta),
$$

and postulate the following flow equation for an operator $O$ :

$$
\frac{d O(\sigma, t)}{d t}=\frac{1}{\hbar}[H, O(\sigma, t)] .
$$


The formal solution to the flow equation (3.19) is

$$
O(\sigma, t)=\mathrm{e}^{\frac{1}{\hbar} t H} O(\sigma) \mathrm{e}^{-\frac{1}{\hbar} t H} .
$$

This setup allows us to interpret the model as defined over the cylinder. In particular, we get from (3.18) and (3.19) that $J_{\mathrm{L}}(t+\sigma), G_{\mathrm{L}}^{ \pm}(t+\sigma), L_{\mathrm{L}}(t+$ $\sigma)$ and $J_{\mathrm{R}}(t-\sigma), G_{\mathrm{R}}^{ \pm}(t-\sigma), L_{\mathrm{R}}(t-\sigma)$. Thus we have left and right moving $N=2$ algebras defined over a cylinder.

\section{Classical sigma model}

In this section, we consider the classical supersymmetric non-linear sigma model defined over $S^{1} \times \mathbb{R}$, with Minkowski signature. The Euclidean case can be treated along the same lines.

The supersymmetric $N=(1,1)$ sigma model is defined by the following action functional:

$$
S=\frac{1}{2} \int d \sigma d t d \theta^{-} d \theta^{+} D_{+} \Phi^{\mu} D_{-} \Phi^{\nu} g_{\mu \nu}(\Phi),
$$

where we use the $N=(1,1)$ superfield formalism and $g$ is a metric on the target $M$. The even coordinate $\sigma$ parametrizes the circle $S^{1}$ and $t$ is the time coordinate for $\mathbb{R}$. The pair $\theta^{ \pm}$labels the spinor coordinates. The spinor derivatives $D_{ \pm}$are defined as

$$
D_{ \pm}=\frac{\partial}{\partial \theta^{ \pm}}+\theta^{ \pm}\left(\partial_{0} \pm \partial_{1}\right), \quad D_{ \pm}^{2}=\partial_{0} \pm \partial_{1},
$$

where $\partial_{0} \equiv \frac{\partial}{\partial t}$ and $\partial_{1} \equiv \frac{\partial}{\partial \sigma}$. This action is invariant under $N=(1,1)$ superconformal transformations. If the target manifold $M$ is Kähler then the action (4.1) is invariant under $N=(2,2)$ superconformal symmetry and in particular it is invariant under the following transformations:

$$
\delta \Phi^{\mu}=\epsilon^{+} D_{+} \Phi^{\nu} I_{\nu}^{\mu}(\Phi)+\epsilon^{-} D_{-} \Phi^{\nu} I_{\nu}^{\mu}(\Phi),
$$

where $\epsilon^{ \pm}$are odd functions subject to the condition $D_{ \pm} \epsilon^{\mp}=0$, and $I$ is the complex structure on the target $M$.

We would like to define the Hamiltonian formalism for the model and discuss the Hamiltonian realization of the symmetries of the action (4.1). In 
going to Hamiltonian formalism we would like to get rid of one odd $\theta$. Let us introduce new odd coordinates as follows:

$$
\theta^{+}=\frac{1}{\sqrt{2}}\left(\theta_{0}+\theta_{1}\right), \quad \theta^{-}=\frac{1}{\mathrm{i} \sqrt{2}}\left(\theta_{0}-\theta_{1}\right),
$$

together with odd derivatives

$$
D_{+}=\frac{1}{\sqrt{2}}\left(D_{0}+D_{1}\right), \quad D_{-}=\frac{1}{\mathrm{i} \sqrt{2}}\left(D_{0}-D_{1}\right),
$$

which satisfy $D_{0}^{2}=\partial_{1}, D_{1}^{2}=\partial_{1}$, and $D_{1} D_{0}+D_{0} D_{1}=2 \partial_{0}$. In order to integrate out $\theta_{0}$ we introduce new superfields:

$$
\phi^{\mu}=\left.\Phi^{\mu}\right|_{\theta_{0}=0}, S_{\mu}=\left.g_{\mu \nu} D_{0} \Phi^{\nu}\right|_{\theta_{0}=0},
$$

and from now on $D_{1}=\left.D_{1}\right|_{\theta_{0}=0}$. After performing $\theta_{0}$-integration, the action (4.1) becomes

$$
S=\int d t d \sigma d \theta_{1}\left(S_{\mu} \partial_{0} \phi^{\mu}-\frac{1}{2} \mathcal{H}\right)
$$

where

$$
\mathcal{H}=\partial_{1} \phi^{\mu} D_{1} \phi^{\nu} g_{\mu \nu}+g^{\mu \nu} S_{\mu} D_{1} S_{\nu}+S_{\rho} D_{1} \phi^{\gamma} S_{\lambda} g^{\nu \lambda} \Gamma^{\rho}{ }_{\gamma \nu} .
$$

Thus we can conclude that the sigma model phase space corresponds to a cotangent bundle $T^{*} \mathcal{L} M$ to a superloop space $\mathcal{L} M=\left\{S^{1 \mid 1} \rightarrow M\right\}$ equipped with the natural symplectic structure

$$
\int d \sigma d \theta_{1} \delta S_{\mu} \wedge \delta \phi^{\mu}
$$

Here $\theta_{1}$ transforms as a section of the square root of the canonical bundle over $S^{1}$. Thus the space of functionals on $T^{*} \mathcal{L} M$ is equipped with a superPoisson bracket $\{$,$\} generated by the relation$

$$
\left\{\phi^{\mu}\left(\sigma, \theta_{1}\right), S_{\nu}\left(\sigma^{\prime}, \theta_{1}^{\prime}\right)\right\}=\delta_{\nu}^{\mu} \delta\left(\sigma-\sigma^{\prime}\right) \delta\left(\theta_{1}-\theta_{1}^{\prime}\right) .
$$

From (4.7) and (4.8) the Hamiltonian is:

$$
H=\frac{1}{2} \int d \sigma d \theta_{1} \mathcal{H}
$$


and the sigma model equation of motions are

$$
\begin{aligned}
\frac{\partial \phi^{\mu}}{\partial t}= & \left\{H, \phi^{\mu}\right\}=g^{\mu \nu} D_{1} S_{\nu}-g^{\mu \sigma} \Gamma_{\sigma \nu}^{\lambda} D_{1} \phi^{\nu} S_{\lambda} \\
\frac{\partial S_{\mu}}{\partial t}= & \left\{H, S_{\mu}\right\}=g_{\mu \rho} \partial_{1} D_{1} \phi^{\rho}+\partial_{1} \phi^{\nu} D_{1} \phi^{\rho} \Gamma_{\mu \nu \rho}+S_{\nu} D_{1} S_{\rho} g^{\rho \lambda} \Gamma_{\lambda \mu}^{\nu} \\
& +\frac{1}{2} S_{\nu} D_{1} \phi^{\rho} S_{\lambda}\left(\partial_{\rho}\left(g^{\lambda \gamma} \Gamma_{\mu \gamma}^{\nu}\right)-\partial_{\mu}\left(g^{\lambda \gamma} \Gamma_{\rho \gamma}^{\nu}\right)\right)
\end{aligned}
$$

where $\partial_{\rho}$ and $\partial_{\mu}$ in the last equation refer to derivatives along the target coordinates. Equations (4.11) are equivalent to the equations which follow from the variational principle for (4.1).

Next, let us discuss how the symmetries of the action functional (4.1) are realized in the Hamiltonian formalism. It is a tedious but straightforward computation to check that (4.3) are realized in the Hamiltonian formalism by the following generators:

$$
\begin{aligned}
& \mathcal{J}_{1}\left(\epsilon_{1}\right)=\int d \sigma d \theta_{1} \epsilon_{1}\left(\sigma, \theta_{1}\right) I_{\nu}^{\mu} D_{1} \phi^{\nu} S_{\mu} \\
& \mathcal{J}_{2}\left(\epsilon_{2}\right)=\frac{1}{2} \int d \sigma d \theta_{1} \epsilon_{2}(\sigma, \theta)\left(\omega^{\mu \nu} S_{\mu} S_{\nu}-\omega_{\mu \nu} D_{1} \phi^{\mu} D_{1} \phi^{\nu}\right),
\end{aligned}
$$

where $I$ is complex structure and $\omega=g I$ is Kähler form. The parameters are related as follows:

$$
\epsilon_{1}=\frac{1}{\sqrt{2}}\left(\mathrm{i} \epsilon_{-}+\epsilon_{+}\right), \quad \epsilon_{2}=\frac{1}{\sqrt{2}}\left(\mathrm{i} \epsilon_{-}-\epsilon_{+}\right),
$$

where $\epsilon_{ \pm}$are evaluated at $t=0$. The remaining generators of $N=(2,2)$ superconformal symmetry can be calculated by computing the Poisson brackets between different combinations of $\mathcal{J}_{1}$ and $\mathcal{J}_{2}$. In addition to (4.12) and (4.13) we obtain

$$
\mathcal{P}\left(a_{1}\right)=\int d \sigma d \theta_{1} a_{1}\left(\sigma, \theta_{1}\right)\left(D_{1} \phi^{\mu} D_{1} S_{\mu}+\partial_{1} \phi^{\mu} S_{\mu}\right),
$$

and

$$
\begin{aligned}
\mathcal{H}\left(a_{2}\right)= & \int d \sigma d \theta_{1} a_{2}\left(\sigma, \theta_{1}\right) \\
& \times\left(\partial_{1} \phi^{\mu} D_{1} \phi^{\nu} g_{\mu \nu}+g^{\mu \nu} S_{\mu} D_{1} S_{\nu}+S_{\rho} D_{1} \phi^{\gamma} S_{\lambda} g^{\nu \lambda} \Gamma_{\gamma \nu}^{\rho}\right)
\end{aligned}
$$

thus obtaining the Hamiltonian realization of the $N=(2,2)$ superconformal symmetries of the action (4.1). 
In order to compare these results with the expressions of the previous section, we perform a change of coordinates. Since we are in the classical setup, we can easily change coordinates even at the Lagrangian level. In particular, we perform the transformation $\xi=\mathrm{e}^{\mathrm{i} \sigma},(\mathrm{i} \xi)^{-1 / 2} \theta=\theta_{1}$, which imply

$$
(\mathrm{i} \xi)^{1 / 2} D=D_{1}, \quad(\mathrm{i} \xi)^{1 / 2} d \theta=d \theta_{1}, \quad(\mathrm{i} \xi)^{1 / 2} S_{\mu}(\xi, \theta)=S_{\mu}\left(\sigma, \theta_{1}\right),
$$

where we now use the notations from the previous two sections. Now, one can easily see that all Hamiltonian generators of $N=(2,2)$ superconformal symmetry derived from the action principle are mapped directly to the expressions from the previous section, modulo $\hbar$-terms,

$$
\begin{aligned}
\mathcal{P}(\xi, \theta) & =D \phi^{\mu} D S_{\mu}+\partial \phi^{\mu} S_{\mu}, \\
\mathcal{J}_{1}(\xi, \theta) & =\left(I_{\nu}^{\mu} D \phi^{\nu}\right) S_{\mu} \\
\mathcal{J}_{2}(\xi, \theta) & =\frac{1}{2}\left(\omega^{\mu \nu} S_{\mu} S_{\nu}-\omega_{\mu \nu} D \phi^{\mu} D \phi^{\nu}\right) \\
\mathcal{H}(\xi, \theta) & =\partial \phi^{\mu} D \phi^{\nu} g_{\mu \nu}+g^{\mu \nu} S_{\mu} D S_{\nu}+S_{\rho} D \phi^{\sigma} S_{\lambda} g^{\nu \lambda} \Gamma_{\sigma \nu}^{\rho} .
\end{aligned}
$$

In particular, the Hamiltonian becomes

$$
H=\frac{\mathrm{i}}{2} \int d \xi d \theta \xi\left(\partial \phi^{\mu} D \phi^{\nu} g_{\mu \nu}+g^{\mu \nu} S_{\mu} D S_{\nu}+S_{\rho} D \phi^{\sigma} S_{\lambda} g^{\nu \lambda} \Gamma_{\sigma \nu}^{\rho}\right),
$$

which is the same as in (3.18). The conventions for the Poisson brackets and the brackets for the above classical generators can be found in the appendix, see (A.21) to (A.28).

\section{Interpretation}

In this section, we combine the results from the two previous sections. We offer both physical and mathematical interpretations of these results.

\subsection{Physical aspects}

In Section 4 we analyzed, in the Hamiltonian formalism, the classical supersymmetric non-linear sigma model with a Kähler manifold as target. Its phase space is defined as the cotangent bundle to a superloop space $T^{*} \mathcal{L} M$. The canonical Poisson bracket is given in local coordinates by the following 
expression:

$$
\left\{\phi^{\mu}(\xi, \theta), S_{\nu}\left(\xi^{\prime}, \theta^{\prime}\right)\right\}=\delta_{\nu}^{\mu} \delta\left(\xi-\xi^{\prime}\right) \delta\left(\theta-\theta^{\prime}\right) .
$$

Moreover, we have found the generators $\left(\mathcal{P}, \mathcal{J}_{1}, \mathcal{J}_{2}, \mathcal{H}\right)$ of $N=(2,2)$ superconformal symmetries in the Hamiltonian formalism and calculated their Poisson brackets (A.21) to (A.28). These generators correspond to the Hamiltonian realization of the superconformal symmetries of the action (4.1). One trivial, but important, aspect is that coordinate changes $\tilde{x}^{\mu}=$ $g^{\mu}(x)$ (with inverse $x^{\mu}=f^{\mu}(\tilde{x})$ ) can be mapped to symplectomorphisms of (5.1):

$$
\tilde{\phi}^{\mu}=g^{\mu}(\phi), \quad \tilde{S}_{\mu}=\frac{\partial f^{\mu}}{\partial \tilde{\phi}^{\nu}}(g(\phi)) S_{\nu} .
$$

Thus it is very easy to construct local expressions which are diffeomorphism invariant and calculate their Poisson brackets.

In Section 3 we considered the $\operatorname{CDR} \Omega_{\hbar}^{\text {ch }}(M)$ on a Calabi-Yau manifold $M . \Omega_{\hbar}^{\text {ch }}(M)$ is a sheaf of SUSY vertex algebras which are defined locally by the commutator:

$$
\left[\phi^{\mu}(\xi, \theta), S_{\nu}\left(\xi^{\prime}, \theta^{\prime}\right)\right]=\hbar \delta_{\nu}^{\mu} \delta\left(\xi-\xi^{\prime}\right) \delta\left(\theta-\theta^{\prime}\right)
$$

which can be understood as a canonical quantization ${ }^{4}$ of the structure (5.1). As in any operatorial quantization, we have to deal with the ordering problem. In CDR, we choose the standard normal ordering for all operators. Once we choose the ordering, we can look at the normal ordered version of (5.2) (see (2.9)) and ask if it respects the commutator (5.3). As it stands, this is a complicated issue and it requires additional care. CDR gives meaning and a solution to this question. Thus, CDR provides a prescription by which to perform a canonical quantization of the non-linear sigma model. Next, we look at the normally ordered expressions for the classical $\left(\mathcal{P}, \mathcal{J}_{1}, \mathcal{J}_{2}, \mathcal{H}\right)$ and it turns out that $\mathcal{P}$ and $\mathcal{J}_{1}$ are not well-defined under "quantum diffeomorphisms" unless they are corrected by $\hbar$-terms. In order to be able to do this we require that $M$ is a Calabi-Yau manifold. Once we have well-defined operators, we can calculate the operator algebra they generate, which turns out to be a central extension of the $N=(2,2)$ superconformal algebra.

Therefore, CDR suggests to perform a canonical quantization locally on $M$ and then glue each patch in an appropriate sense. Indeed, if $M$ is an affine space with its standard flat metric, then the canonical quantization of the sigma model can be carried out completely. The reader may consult the next section for explicit formulas. In fact, the free $\beta \gamma b c$-system (with

\footnotetext{
${ }^{4}$ Here we miss the factor "i" which is important for unitarity issues. In the present consideration, we proceed formally and ignore the hermiticity of the operators.
} 
an appropriate choice of Hamiltonian) can be interpreted as the canonical quantization of the sigma model with flat metric over an affine space.

The set of global sections $V_{\hbar}:=\Gamma\left(M, \Omega_{\hbar}^{\text {ch }}(M)\right)$ is a family of vertex algebras associated to the manifold $M$ (depending on the parameter $\hbar$ ). The operators from $V_{\hbar}$ are interpreted as operators of the quantum sigma model in the Schrödinger picture (i.e., the operators are taken at fixed time, say $t=0)$. If we choose a Hamiltonian $H \in V_{\hbar}$, and postulate the flow equation (3.19), then we can work in the Heisenberg picture (3.20). From this point of view, CDR does not carry any dynamical information unless a Hamiltonian is introduced. This is consistent for example with results from [9], where the authors derive CDR as the phase space for a suitable limit of the sigma-model.

The structures which we find in CDR can be equivalently found with the path integral approach. CDR carries information about the equal time commutators, while the path integral calculates the time ordered correlators:

$$
\left\langle T\left(O_{1}(\xi, t), O_{2}\left(\xi^{\prime}, t^{\prime}\right)\right)\right\rangle=\int D \phi D S O_{1}(\xi, t) O_{2}\left(\xi^{\prime}, t^{\prime}\right) \mathrm{e}^{\frac{1}{\hbar}\left(\int d t d \xi d \theta S_{\mu} \partial_{t} \phi^{\mu}-\int d t H\right)}
$$

where $T$ stands for the time ordering. In order to calculate equal time commutators, we have to use the Bjorken-Johnson-Low prescription ${ }^{5}$

$$
\left\langle\left[O_{1}(\xi, t), O_{2}\left(\xi^{\prime}, t\right)\right]\right\rangle=\left(\lim _{t \rightarrow t^{\prime}+0}-\lim _{t \rightarrow t^{\prime}-0}\right)\left\langle T\left(O_{1}(\xi, t), O_{2}\left(\xi^{\prime}, t^{\prime}\right)\right)\right\rangle
$$

The result of this calculation is independent from the Hamiltonian $H$. We can use this point splitting procedure to define our local operators and study their properties under diffeomorphisms of the target manifold.

Let us a make a few concluding remarks. Our manipulations are formal and it is open to further investigation how the Hamiltonian interpretation of CDR can help us to understand the quantum sigma model. Especially one may need extra input for the analysis of the solutions of the flow equation, for example in order to make sense of some analytical issues. Eventually, we would like to understand how to calculate and study the properties of the non-equal time correlators in the full quantum theory.

In the present considerations $M$ is assumed to be a simply connected manifold. If $M$ is not simply connected then the phase space $T^{*} \mathcal{L} M$ is not

\footnotetext{
${ }^{5}$ It is important that we do not have a boundary in this field theory. The canonical relations may be modified by boundary contributions, e.g., see, [1].
} 
connected and thus we have to face further complications in its quantization. Moreover, even if $M$ is simply connected, $\mathcal{L} M$ may fail to be simply connected. In this situation, one can replace $M$ by a $U(1)$ gerbe with connection on $M$, or more generally by any Courant algebroid on $M$. CDR is replaced in this setup by the sheaves of SUSY vertex algebras studied in $[11,12]$.

\subsection{Mathematical aspects}

In this section, we briefly review the quasiclassical limit of the CDR. For a thorough introduction to Poisson vertex algebras as limits of vertex algebras and the corresponding Hamiltonian equations we refer the reader to [5] (see also $[6, \S 16.2])$. For an extensive study of sheaves of Poisson vertex algebras and their relation to CDR see [17].

Suppose we are given a family of vertex algebra structures $V_{\hbar}$ on the same vector space $V$. That is, we have a collection of operations $A_{(n, \hbar)} B \in V$ for all $A, B \in V$. We may think of the family $V_{\hbar}$ as a vertex algebra over the ring of power series $\mathbb{C}[[\hbar]]$ instead of simply $\mathbb{C}$. Suppose moreover that in the limit $V_{0}=\lim _{\hbar \rightarrow 0} V_{\hbar}:=V_{\hbar} / \hbar V_{\hbar}$, the vertex algebra becomes commutative, that is, all the fields

$$
Y_{\hbar}(A, \xi)=\sum_{n \in \mathbb{Z}} \xi^{-1-n} A_{(n, \hbar)},
$$

commute modulo $\hbar$-terms. Equivalently, it follows from (2.2) that all the products $A_{(j, \hbar)} B, j \geq 0$, vanish modulo $\hbar$-terms, and moreover, the normally ordered product is commutative modulo $\hbar$-terms.

Note that this in particular says that the $\lambda$-bracket vanishes modulo $\hbar$, hence we can rescale our commutators on $V_{0}$ to define a new operation:

$$
\left\{A_{\lambda} B\right\}=\lim _{\hbar \rightarrow 0} \frac{1}{\hbar}\left[A_{\lambda} B\right]_{\hbar},
$$

or, equivalently

$$
\left\{A(\xi), B\left(\xi^{\prime}\right)\right\}=\lim _{\hbar \rightarrow 0} \frac{1}{\hbar} \sum_{j \geq 0} \frac{1}{j !}\left(A_{(j, \hbar)} B\right)\left(\xi^{\prime}\right) \partial_{\xi^{\prime}}^{j} \delta\left(\xi-\xi^{\prime}\right) .
$$

$V_{0}$ with its commutative product

$$
A \cdot B:=\lim _{\hbar \rightarrow 0} A_{(-1, \hbar)} B,
$$


its Poisson $\lambda$-bracket (5.6), and its derivation

$$
\partial A:=\lim _{\hbar \rightarrow 0} A_{(-2, \hbar)}|0\rangle,
$$

acquires an extra structure known as a Poisson vertex algebra and it is called the quasiclassical limit of the family $V_{\hbar}$.

Performing computations on $V_{0}$ is generally much simpler than performing computations on $V_{\hbar}$. For example, the bracket $\{\lambda\}$ and the product $\cdot$ satisfy a simple Leibniz rule on $V_{0}$ :

$$
\left\{A_{\lambda} B \cdot C\right\}=\left\{A_{\lambda} B\right\} \cdot C+B \cdot\left\{A_{\lambda} C\right\}
$$

while the corresponding relation on $V_{\hbar}-$ known as the non-commutative Wick formula, reads (we omit the $\hbar$ in the operations):

$$
\left[A_{\lambda}: B C:\right]=:\left[A_{\lambda} B\right] C:+: B\left[A_{\lambda} C\right]:+\int_{0}^{\lambda}\left[\left[A_{\lambda} B\right]_{\gamma} C\right] d \gamma
$$

Note that the integral term, under our hypothesis, vanishes of order $\hbar^{2}$ since it is a double commutator. In particular, this quantum correction disappears in the limit $\hbar \rightarrow 0$ even after rescaling the OPE as in (5.6). It is in this sense that one usually refers to Poisson vertex algebras as "vertex algebras with the quantum corrections removed".

In Section 2 we constructed a family of sheaves of vertex algebras $\Omega_{\hbar}^{\text {ch }}(M)$ on a manifold $M$. Taking global sections one obtains a family of vertex algebras $V_{\hbar}:=\Gamma\left(M, \Omega_{\hbar}^{\text {ch }}(M)\right)$. And finally, we obtain a Poisson vertex algebra by the above limiting procedure: $V_{0}:=\lim _{\hbar \rightarrow 0} V_{\hbar}$. In fact, one can first take the limit locally, to obtain a sheaf $\mathcal{V}_{0}$ of Poisson vertex algebras, and then taking global sections we find $V_{0} \simeq \Gamma\left(M, \mathcal{V}_{0}\right)$. Indeed, in a local coordinate chart $\left\{x_{\mu}\right\}, \mathcal{V}_{0}$ is generated by fields (cf. (2.5) and (2.6)) $\left(\beta_{\mu}, \gamma^{\mu}\right)$ with their Poisson bracket:

$$
\left\{\beta_{\mu}(\xi), \gamma^{\nu}\left(\xi^{\prime}\right)\right\}=\delta_{\mu}^{\nu} \delta\left(\xi-\xi^{\prime}\right)
$$

or equivalently

$$
\left\{\beta_{\mu_{\lambda}} \gamma^{\nu}\right\}=\delta_{\mu}^{\nu}
$$

together with the fermionic fields $\left(b_{\mu}, c^{\mu}\right)$ satisfying the Poisson bracket: ${ }^{6}$

$$
\left\{b_{\mu}(\xi), c^{\nu}\left(\xi^{\prime}\right)\right\}=\delta_{\mu}^{\nu} \delta\left(\xi-\xi^{\prime}\right), \quad \text { or } \quad\left\{b_{\mu_{\lambda}} c^{\nu}\right\}=\delta_{\mu}^{\nu}
$$

One obtains other fields of $\mathcal{V}_{0}$ using the commutative product $\cdot$ and the derivation $\partial$.

\footnotetext{
${ }^{6}$ This is a $\mathbb{Z}_{2}$ graded bracket.
} 
The above said can be easily generalized to the case when the fields depend on an extra odd coordinate $\theta$, namely, when we have a family $V_{\hbar}$ of SUSY vertex algebras, such that it becomes commutative in the limit $V_{0}=\lim _{\hbar \rightarrow 0} V_{\hbar}$. Again we find that $\mathcal{V}_{0}$ is generated by even superfields $\phi^{\mu}(\xi, \theta)=\gamma^{\mu}(\xi)+\theta c^{\mu}(\xi)$ and odd superfields $S_{\mu}(\xi, \theta)=b_{\mu}(\xi)+\theta \beta_{\mu}(\xi)$ satisfying the super-Poisson bracket:

$$
\left\{\phi^{\nu}(\xi, \theta), S_{\mu}\left(\xi^{\prime}, \theta^{\prime}\right)\right\}=\delta_{\mu}^{\nu} \delta\left(\xi-\xi^{\prime}\right) \delta\left(\theta-\theta^{\prime}\right) .
$$

We see that this coincides with (4.10), thus it is not surprising that formulas computed at the quantum level in CDR, when viewed modulo $\hbar$-terms, that is, at the quasiclassical level, coincide with the formulas of Section 4 .

Under a change of coordinates $\tilde{x}^{\mu}=g^{\mu}(x)$ with its inverse $x^{\mu}=f^{\mu}(\tilde{x})$, the superfields $\phi^{\mu}$ and $S_{\mu}$ transform as in (2.9), but the multiplication is now commutative and associative. In particular, $\mathcal{V}_{0}$ is a (infinite rank) vector bundle on $M$. Note however, that even though the generating sections of $\mathcal{V}_{0}$ transform in a tensorial manner, not all local sections do, as the example of $D S_{\mu}=\left(\partial_{\theta}+\theta \partial_{\xi}\right) S_{\mu}(\xi, \theta)$ shows.

Using the above-mentioned formalism, we can now interpret the results of Section 4 as a classical limit of the equations of Section 3. In fact, the expression for $\mathcal{P}$ in (4.16) defines a field of $V_{0}$, which is now interpreted as the limit of the fields of $V_{\hbar}$ defined by (3.1). Similarly, one obtains all the generators of $N=(2,2)$ superconformal symmetries of the classical sigma model on a Calabi-Yau manifold $M(4.16)$ to (4.19), as the limit of the corresponding sections of CDR on $M$.

Given a Poisson vertex algebra $V_{0}$ one usually considers elements of $V_{0} / \partial V_{0}$ as "local functionals" [5, Remark 6.3]. And for a given local functional $H$, one considers the "Hamiltonian equations"

$$
\dot{u}=\{H, u\}, \quad u \in V_{0} .
$$

In a similar manner, for a given vertex algebra $V$ and an element $H \in V / \partial V$ we can construct the "quantum Hamiltonian equations"

$$
\dot{u}=\frac{1}{\hbar}[H, u], \quad u \in V .
$$

Let $M$ be a Calabi-Yau manifold and let $V_{\hbar}$ be the vertex algebra of global sections of CDR, $V_{0}$ its quasiclassical limit described above. Recall that we have the global section $\mathcal{H}(3.8)$, and this clearly gives rise to an element of 
$V_{0}$ as well. We consider the Hamiltonian

$$
H=\frac{1}{2} \int d \sigma d t \mathcal{H}
$$

For this choice of Hamiltonian in $V_{0}$, Equations (5.14) agree with the equations of motion (4.11), derived in the context of the classical non-linear sigma model. Therefore, we see that the classical limit of the CDR of $M$ together with the choice of Hamiltonian (5.16) recovers the full dynamics of the classical non-linear sigma model on $M$.

Note, however, that since $\mathcal{H}$ is a well-defined section in $V_{\hbar}$ for all $\hbar$, we can use $H$ as a quantum Hamiltonian to write down some quantum equations of motion. We refrain from doing so here since these equations are not enlightening.

Remark 5.1. We have remarked earlier that the section (3.8) was constructed in [11] under the assumption that $M$ is Calabi-Yau. Even though we conjecture that this section is well defined on any Riemannian manifold $M$, for the purposes of this section, we only need its zero mode $H$. It is straightforward to check that $H$ is well defined regardless of whether $\mathcal{H}$ is.

Remark 5.2. Note that by solving formally (5.15) for each $u \in V$ and by using the state-field correspondence $Y$ of $V$, we can associate a "field of two variables" 7

$$
u \mapsto Y(u, \sigma, t):=Y(u(t), \sigma) .
$$

Following [6] we can formally use the Virasoro $L_{\mathrm{L}}$ (resp. $L_{\mathrm{R}}$ ) from Section 3 to see how these "fields" change with respect to changes in the coordinate $\sigma+t$ (resp. $\sigma-t$ ), but a priori we do not know how to deal with general coordinates in the worldsheet. The appropriate algebraic axioms satisfied by the fields (5.17) will be studied elsewhere.

\section{$6 \quad$ Free field examples}

In this section we consider the free field examples and for simplicity we set $\hbar=1$. We remind some standard facts about $\beta \gamma$ - and $b c$-systems and also stress some aspects of these systems in light of our previous discussion. Although we here repeat explicitly some of the formulas from Section 3 we find this discussion instructive and clarifying.

\footnotetext{
${ }^{7}$ Here we obviate the odd coordinates for simplicity.
} 


\subsection{Free boson}

Consider a single $\beta \gamma$-system defined by the bracket

$$
\left[\beta_{\lambda} \gamma\right]=1
$$

where we use the $\lambda$-bracket notation, see Section 2. The Virasoro field is defined as

$$
L=\beta \partial \gamma
$$

which satisfies the Virasoro algebra:

$$
\left[L_{\lambda} L\right]=(2 \lambda+\partial) L+\frac{2 \lambda^{3}}{12}
$$

with central charge 2 . With respect to this $L, \beta$ is a primary field of conformal weight 1 and $\gamma$ is of conformal weight 0 ,

$$
\gamma(\xi)=\sum_{n \in \mathbb{Z}} \gamma_{n} \xi^{-n}, \quad \beta(\xi)=\sum_{n \in \mathbb{Z}} \beta_{n} \xi^{-n-1}
$$

The vacuum is annihilated by $\beta_{n}(n \geq 0)$ and $\gamma_{n}(n>0)$, but $\gamma_{0}$ is considered to be a creator. The field $L$ can be split into two parts as follows:

$$
L_{\mathrm{L}}=\frac{1}{2}\left[\beta \partial \gamma+\frac{1}{2}\left(\beta^{2}+(\partial \gamma)^{2}\right)\right], \quad L_{\mathrm{R}}=\frac{1}{2}\left[\beta \partial \gamma-\frac{1}{2}\left(\beta^{2}+(\partial \gamma)^{2}\right)\right]
$$

$L_{\mathrm{L}}$ and $L_{\mathrm{R}}$ give rise to two commuting copies of the Virasoro algebra with central charge $c=1$ each:

$$
\begin{aligned}
& {\left[L_{\mathrm{L}_{\lambda}} L_{\mathrm{L}}\right]=(2 \lambda+\partial) L_{\mathrm{L}}+\frac{\lambda^{3}}{12},} \\
& {\left[L_{\mathrm{R}_{\lambda}} L_{\mathrm{R}}\right]=(2 \lambda+\partial) L_{\mathrm{R}}+\frac{\lambda^{3}}{12},} \\
& {\left[L_{\mathrm{L}_{\lambda}} L_{\mathrm{R}}\right]=0 .}
\end{aligned}
$$

Neither $\beta$ nor $\gamma$ are primary fields with respect to $L_{\mathrm{L}}$ and $L_{\mathrm{R}}$. However the field $\frac{1}{\sqrt{2}}(\beta+\partial \gamma)$ (resp. $\left.\frac{1}{\sqrt{2}}(\beta-\partial \gamma)\right)$ is primary of conformal weight 1 with 
respect to $L_{\mathrm{L}}\left(\right.$ resp. $\left.L_{\mathrm{R}}\right)$. Indeed, introducing new fields

$$
\alpha_{ \pm}=\frac{\beta \pm \partial \gamma}{\sqrt{2}}
$$

which satisfy the following brackets:

$$
\left[\alpha_{ \pm \lambda} \alpha_{ \pm}\right]= \pm \lambda, \quad\left[\alpha_{ \pm \lambda} \alpha_{\mp}\right]=0
$$

the Virasoro fields $L_{\mathrm{L} / \mathrm{R}}$ can be written as

$$
L_{\mathrm{L}}=\frac{1}{2} \alpha_{+}^{2}, \quad L_{\mathrm{R}}=-\frac{1}{2} \alpha_{-}^{2}
$$

These are just formal observations about the vertex algebra of the $\beta \gamma$ system.

Next, let us introduce a new parameter $t$, and a $t$-dependence on our fields by means of flow equations. We choose the Hamiltonian to be

$$
H=\mathrm{i} \int d \xi \xi\left(L_{\mathrm{L}}-L_{\mathrm{R}}\right)=\frac{\mathrm{i}}{2} \int d \xi \xi\left(\beta^{2}+(\partial \gamma)^{2}\right)
$$

or equivalently, in the $\sigma$-coordinates $\xi=\mathrm{e}^{\mathrm{i} \sigma}$ :

$$
H=\frac{1}{2} \int d \sigma\left(\beta^{2}+\left(\partial_{\sigma} \gamma\right)^{2}\right)
$$

In performing this coordinate change we have used that $\beta$ is of conformal weight one and $\gamma$ is of conformal weight zero, and we use the "diagonal Virasoro" $L=L_{\mathrm{L}}+L_{\mathrm{R}}$ to perform changes of coordinates. The flow equations are

$$
\frac{d \gamma}{d t}=\beta, \quad \frac{d \beta}{d t}=\partial_{\sigma}^{2} \gamma
$$

and these are exactly the Hamiltonian equations for the free boson theory. The solution of (6.12) is given by the following expression:

$$
\gamma(\sigma, t)=\gamma_{0}+\beta_{0} t+\sum_{n \neq 0} \frac{1}{2}\left[\left(\gamma_{n}+\frac{\mathrm{i}}{n} \beta_{n}\right) \mathrm{e}^{-\mathrm{i} n(\sigma+t)}+\left(\gamma_{n}-\frac{\mathrm{i}}{n} \beta_{n}\right) \mathrm{e}^{-\mathrm{i} n(\sigma-t)}\right]
$$

Thus, the $\beta \gamma$-system with an appropriate choice of Hamiltonian can be identified with the standard free boson theory, which contains both chiral and anti-chiral sectors. Among the solutions to the flow equation, the field $\alpha_{+}$ corresponds to the left moving (chiral) sector, while $\alpha_{-}$to the right moving 
(anti-chiral) sector. It is crucial for the identification with the free boson that $\gamma_{0}$ is a creator so that we have states

$$
\mathrm{e}^{\mathrm{i} k \gamma_{0}}|0\rangle \equiv|k\rangle, \quad \beta_{0}|k\rangle=\mathrm{i} k|k\rangle, \quad k \in \mathbb{R} .
$$

Let us finish with a simple but important comment. If we choose a different Hamiltonian, then we obtain different flow equations and a different system. For example, if we consider the Hamiltonian

$$
H=\int d \sigma \beta \partial_{\sigma} \gamma
$$

the flow equations are

$$
\frac{d \gamma}{d t}=\partial_{\sigma} \gamma, \quad \frac{d \beta}{d t}=\partial_{\sigma} \beta,
$$

which is just the standard chiral $\beta \gamma$-system.

\subsection{Free fermion}

Next, we consider the super vertex algebra of the free $b c$-system, which is generated by two odd fields $b$ and $c$ satisfying the following bracket:

$$
\left[b_{\lambda} c\right]=1 .
$$

The field

$$
L=\frac{1}{2}((\partial c) b+(\partial b) c),
$$

gives rise to a Virasoro algebra of central charge 1. With respect to $L, c$ and $b$ are primary fields of conformal weight $1 / 2$ :

$$
c(\xi)=\sum_{n \in \mathbb{Z}+1 / 2} c_{n} \xi^{-n-\frac{1}{2}}, \quad b(\xi)=\sum_{n \in \mathbb{Z}+1 / 2} b_{n} \xi^{-n-\frac{1}{2}} .
$$

The vacuum is annihilated by $b_{n}$ and $c_{n}(n>0)$ and the rest of the modes are creators. $L$ can be split into two commuting parts:

$$
L_{\mathrm{L} / \mathrm{R}}=\frac{1}{4}((\partial c) b+(\partial b) c \pm(\partial c) c \pm(\partial b) b),
$$


such that each $L_{\mathrm{L} / \mathrm{R}}$ generate a Virasoro vertex algebra with central charge $\frac{1}{2}$ and they mutually commute. Introducing the new fields

$$
\psi_{+}=\frac{b+c}{\sqrt{2}}, \quad \psi_{-}=\frac{b-c}{\sqrt{2}},
$$

satisfying the brackets

$$
\left[\psi_{ \pm_{\lambda}} \psi_{ \pm}\right]= \pm 1, \quad\left[\psi_{ \pm_{\lambda}} \psi_{\mp}\right]=0,
$$

we rewrite $L_{\mathrm{L} / \mathrm{R}}$ as

$$
L_{\mathrm{L}}=\frac{1}{2}\left(\partial \psi_{+}\right) \psi_{+}, \quad L_{\mathrm{R}}=-\frac{1}{2}\left(\partial \psi_{-}\right) \psi_{-} .
$$

Introducing the Hamiltonian

$$
\begin{aligned}
H & =\mathrm{i} \int d \xi \xi\left(L_{\mathrm{L}}-L_{\mathrm{R}}\right)=\frac{\mathrm{i}}{2} \int d \xi \xi((\partial c) c+(\partial b) b) \\
& =\frac{1}{2} \int d \sigma\left(\left(\partial_{\sigma} c\right) c+\left(\partial_{\sigma} b\right) b\right)
\end{aligned}
$$

the flow equations imply

$$
\frac{d \psi_{ \pm}}{d t} \mp \partial_{\sigma} \psi_{ \pm}=0
$$

and we can recognize these as the left and right moving parts of the standard free periodic fermion system on the cylinder.

\section{3 $\quad N=1$ supersymmetry}

We can combine the $\beta \gamma$ - and $b c$-systems from the previous subsections. The fields

$$
L=\beta \partial \gamma+\frac{1}{2}((\partial c) b+(\partial b) c), \quad G=c \beta+(\partial \gamma) b,
$$

give rise to the $N=1$ superconformal algebra with central charge 3 :

$$
\begin{aligned}
{\left[L_{\lambda} L\right] } & =(2 \lambda+\partial) L+\frac{3 \lambda^{3}}{12}, \\
{\left[L_{\lambda} G\right] } & =\left(\partial+\frac{3}{2} \lambda\right) G, \\
{\left[G_{\lambda} G\right] } & =2 L+\lambda^{2} .
\end{aligned}
$$


Introducing the superfields $\phi=\gamma+\theta c$ and $S=b+\theta \beta$ we have (cf. (3.1)):

$$
\mathcal{P}=G+2 \theta L=D \phi D S+(\partial \phi) S .
$$

With respect to $L, \phi$ is a primary field of conformal weight 0 and $S$ is primary of conformal weight $1 / 2$.

We can split $L$ and $G$ into two copies,

$$
\begin{aligned}
L_{\mathrm{L} / \mathrm{R}} & =\frac{1}{2}\left(\beta \partial \gamma \pm \frac{1}{2}\left(\beta^{2}+(\partial \gamma)^{2}\right)+\frac{1}{2}((\partial c) b+(\partial b) c \pm(\partial c) c \pm(\partial b) b)\right) \\
G_{\mathrm{L} / \mathrm{R}} & =\frac{1}{2}(c \beta+(\partial \gamma) b \pm \beta b \pm(\partial \gamma) c)
\end{aligned}
$$

of commuting $N=1$ superconformal algebras with central charge $3 / 2$ each. Using superfields we can combine

$$
G_{\mathrm{L} / \mathrm{R}}+2 \theta L_{\mathrm{L} / \mathrm{R}}=\frac{1}{2}(D \phi D S+\partial \phi S \pm \partial \phi D \phi \pm(D S) S) .
$$

It is important to stress that the fields $\phi$ and $S$ are not primary with respect to $L_{\mathrm{L} / \mathrm{R}}$, but rather their sum $L=L_{\mathrm{L}}+L_{\mathrm{R}}$. The Hamiltonian is just the sum of the expressions (6.10) and (6.24) and the corresponding flow equations realize the free boson-fermion system.

We can consider $d$ copies of the $\beta \gamma b c$-system. The corresponding $N=1$ superconformal algebra of central charge $3 d$ is generated by

$$
\begin{aligned}
L & =\left(\partial \gamma^{\mu}\right) \beta_{\mu}+\frac{1}{2}\left(\left(\partial b_{\mu}\right) c^{\mu}+\left(\partial c^{\mu}\right) b_{\mu}\right) \\
G & =\left(\partial \gamma^{\mu}\right) b_{\mu}+\left(c^{\mu}\right) \beta_{\mu} .
\end{aligned}
$$

For a constant $d \times d$ symmetric non-degenerate matrix $g_{\mu \nu}$, we define the fields

$$
\begin{array}{ccrl}
\alpha_{+}^{\mu}=\frac{g^{\mu \nu} \beta_{\nu}+\partial \gamma^{\mu}}{\sqrt{2}}, & \alpha_{-}^{\mu}=\frac{g^{\mu \nu} \beta_{\nu}-\partial \gamma^{\mu}}{\sqrt{2}}, \\
\psi_{+}^{\mu}=\frac{g^{\mu \nu} b_{\nu}+c^{\mu}}{\sqrt{2}}, & \psi_{-}^{\mu}=\frac{g^{\mu \nu} b_{\nu}-c^{\mu}}{\sqrt{2}}
\end{array}
$$

with brackets

$$
\begin{aligned}
{\left[\alpha_{ \pm \lambda}^{\mu} \alpha_{ \pm}^{\nu}\right]= \pm g^{\mu \nu} \lambda, } & {\left[\alpha_{+\lambda}^{\mu} \alpha_{-}^{\nu}\right]=0 } \\
{\left[\psi_{ \pm \lambda}^{\mu} \psi_{ \pm}^{\nu}\right]= \pm g^{\mu \nu}, } & {\left[\psi_{+\lambda}^{\mu} \psi_{-}^{\nu}\right]=0 }
\end{aligned}
$$


The $N=1$ algebra (6.32) can be split into two commuting copies $L=L_{\mathrm{L}}+$ $L_{\mathrm{R}}$ and $G=G_{\mathrm{L}}+G_{\mathrm{R}}[13$, Ex. 5.9a],

$$
\begin{aligned}
L_{\mathrm{L} / \mathrm{R}} & = \pm \frac{1}{2} \alpha_{ \pm}^{\mu} g_{\mu \nu} \alpha_{ \pm}^{\nu} \pm \frac{1}{2} \partial \psi_{ \pm}^{\mu} g_{\mu \nu} \psi_{ \pm}^{\nu}, \\
G_{\mathrm{L} / \mathrm{R}} & = \pm \alpha_{ \pm}^{\mu} g_{\mu \nu} \psi_{ \pm}^{\nu} .
\end{aligned}
$$

Each has central charge $\frac{3}{2} d$. If we choose as Hamiltonian $\mathrm{i}\left(L_{\mathrm{L}}\right)_{0}-\mathrm{i}\left(L_{\mathrm{R}}\right)_{0}$, we obtain the quantization of the sigma model on $\mathbb{R}^{d}$ with metric $g_{\mu \nu}$.

On a general manifold $M$ we can glue together $\beta \gamma b c$-systems according to [16]. When $M$ is orientiable, the generators (6.31) and (6.32) can be glued in a consistent manner (they are the components of (3.1)).

\section{4 $N=2$ supersymmetry}

Next we consider $\mathbb{C}^{n}=\mathbb{R}^{2 n}$ and we follow the notations from Section 3. If we choose a constant symplectic form $\omega$ then the fields $\left(J_{2}, G_{2}^{ \pm}, L_{2}\right)$, with $L_{2}=L$ in (6.31), $G_{2}^{+}+G_{2}^{-}=G$ in (6.32) and

$$
\begin{aligned}
J_{2} & =\frac{\mathrm{i}}{2}\left(\omega^{\mu \nu} b_{\mu} b_{\nu}-\omega_{\mu \nu} c^{\mu} c^{\nu}\right), \\
G_{2}^{-}-G_{2}^{+} & =\mathrm{i}\left(\omega^{\mu \nu} \beta_{\mu} b_{\nu}-\omega_{\mu \nu} \partial \gamma^{\mu} c^{\nu}\right),
\end{aligned}
$$

generate the $N=2$ superconformal algebra with central charge $6 n$. If we choose the standard constant complex structure $I$, then the fields $\left(J_{1}, G_{1}^{ \pm}, L_{1}\right)$ with $L_{1}=L$ in $(6.31), G_{1}^{+}+G_{1}^{-}=G$ in (6.32) and

$$
\begin{aligned}
J_{1} & =\mathrm{i} I_{\nu}^{\mu} c^{\nu} b_{\mu}, \\
G_{1}^{-}-G_{1}^{+} & =\mathrm{i} I_{\nu}^{\mu} \partial \gamma^{\nu} b_{\mu}-\mathrm{i} I_{\nu}^{\mu} c^{\nu} \beta_{\mu},
\end{aligned}
$$

generate the $N=2$ superconformal algebra with central charge $6 n$. If we require that $\omega$ and $I$ are compatible, i.e., we consider the Hermitian metric $g=-\omega I$, then using (6.33) (6.34) we can introduce two commuting copies of the $N=2$ algebra $\left(J_{\mathrm{L} / \mathrm{R}}, G_{\mathrm{L} / \mathrm{R}}^{ \pm}, L_{\mathrm{L} / \mathrm{R}}\right)$ with central charge $\frac{3}{2} d$ each. Together with $L_{\mathrm{L} / \mathrm{R}}$ in (6.37) the other fields are written as [13, Ex. 5.9d]

$$
G_{\mathrm{L} / \mathrm{R}}^{+}= \pm \alpha_{ \pm}^{i} g_{i \bar{j}} \psi_{ \pm}^{\bar{j}}, \quad G_{\mathrm{L} / \mathrm{R}}^{-}= \pm \alpha_{ \pm}^{\bar{i}} g_{\overline{i j}} \psi_{ \pm}^{j}, \quad J_{\mathrm{L} / \mathrm{R}}= \pm \psi_{ \pm}^{\bar{i}} g_{\overline{i j}} \psi_{ \pm}^{j},
$$

where we use holomorphic coordinates $(i, \bar{i})$.

Here one can explicitly see that this is a canonical quantization of the $N=(2,2)$ supersymmetric sigma model with target $\mathbb{C}^{n}$. All operators above 
can be understood in the Schrödinger picture. Introducing the Hamiltonian $\mathrm{i}\left(L_{\mathrm{L}}\right)_{0}-\mathrm{i}\left(L_{\mathrm{R}}\right)_{0}$ we get the correct time dependence.

\subsection{Topological A-and B-twist}

Here we briefly discuss the topological twist of the sigma model which we just discussed. All our formulas are written in the flat $\mathbb{C}^{n}$ case, but they have a straightforward generalization for a Calabi-Yau manifold.

Starting with the left and right moving $N=2$ superconformal algebra $\left(J_{\mathrm{L} / \mathrm{R}}, G_{\mathrm{L} / \mathrm{R}}^{ \pm}, L_{\mathrm{L} / \mathrm{R}}\right)$, we can perform a topological twist. The idea is to redefine the Virasoro field in such a way that the central charge becomes zero. Given the algebras $\left(J_{\mathrm{L} / \mathrm{R}}, G_{\mathrm{L} / \mathrm{R}}^{ \pm}, L_{\mathrm{L} / \mathrm{R}}\right)$ we can define two inequivalent twists, the $\mathrm{A}$-twist and the $\mathrm{B}$-twist. The $\mathrm{A}$-twist is given by

$$
\begin{aligned}
& L_{\mathrm{L}} \rightarrow L_{\mathrm{L}}+\frac{1}{2} \partial J_{\mathrm{L}}, \\
& L_{\mathrm{R}} \rightarrow L_{\mathrm{R}}-\frac{1}{2} \partial J_{\mathrm{R}},
\end{aligned}
$$

and the B-twist by

$$
\begin{aligned}
& L_{\mathrm{L}} \rightarrow L_{\mathrm{L}}+\frac{1}{2} \partial J_{\mathrm{L}}, \\
& L_{\mathrm{R}} \rightarrow L_{\mathrm{R}}+\frac{1}{2} \partial J_{\mathrm{R}} .
\end{aligned}
$$

Using the notations from Section 3 we have $J_{\mathrm{L} / \mathrm{R}}=\left.\frac{\mathrm{i}}{2}\left(\mathcal{J}_{1} \pm \mathcal{J}_{2}\right)\right|_{\theta=0}$, and recall that both $\mathcal{J}_{1}$ and $\mathcal{J}_{2}$ give rise to the same $L=L_{\mathrm{L}}+L_{\mathrm{R}}$. The A-twist corresponds to a twist with respect to $J_{2}=\left.\mathrm{i} \mathcal{J}_{2}\right|_{\theta=0}$, while the Btwist corresponds to a twist with respect to $J_{1}=\left.\mathrm{i} \mathcal{J}_{1}\right|_{\theta=0}$.

Let us perform the A-twist. Consider the $N=2$ algebra $\left(J_{2}, G_{2}^{ \pm}, L_{2}\right)$ and redefine $L_{2} \rightarrow L_{2}+\frac{1}{2} \partial J_{2}$. Defining the new fields:

$$
\begin{aligned}
\psi_{\mu} & =\frac{1}{\sqrt{2}}\left(b_{\mu}-\mathrm{i} \omega_{\mu \nu} c^{\nu}\right), \\
\chi^{\mu} & =\frac{1}{\sqrt{2}}\left(c^{\mu}-\mathrm{i} \omega^{\mu \nu} b_{\nu}\right), \\
\beta_{\mu}^{\prime} & =\frac{\beta_{\mu}-\mathrm{i} \omega_{\mu \nu} \partial \gamma^{\nu}}{\sqrt{2}}, \\
\gamma^{\prime \mu} & =\sqrt{2} \gamma^{\mu},
\end{aligned}
$$

with the brackets

$$
\left[\beta_{\mu_{\lambda}}^{\prime} \gamma^{\prime \nu}\right]=\delta_{\mu}^{\nu}, \quad\left[\chi_{\lambda}^{\mu} \psi_{\nu}\right]=\delta_{\nu}^{\mu}
$$


we obtain a topological $N=2$ algebra $\left(L_{2}, J_{2}, Q_{2}, G_{2}\right)$ :

$$
\begin{aligned}
L_{2} & =\partial \gamma^{\mu} \beta_{\mu}+\partial \chi^{\mu} \psi_{\mu}, \\
J_{2} & =\chi^{\mu} \psi_{\mu}, \\
Q_{2} & =G_{2}^{+}=\chi^{\mu} \beta_{\mu}, \\
G_{2} & =G_{2}^{-}=\psi_{\mu} \partial \gamma^{\mu}-\mathrm{i} \omega^{\mu \nu} \psi_{\mu} \beta_{\nu},
\end{aligned}
$$

where we have omitted ' on $\beta$ and $\gamma$. With respect to this new Virasoro, the fields $\psi_{\mu}$ and $\chi^{\mu}$ have conformal weights 1 and 0 , respectively. Moreover, the odd generators $Q_{2}$ and $G_{2}$ have conformal weights 1 and 2, thus the zero mode of $Q_{2}$ is a BRST operator. Note that the last term in (6.54) can actually be rewritten as

$$
\begin{aligned}
G_{2} & =\psi_{\mu} \partial \gamma^{\mu}+\left[\left(Q_{2}\right)_{0}, V\right], \\
V & =\frac{\mathrm{i} \omega^{\mu \nu} \psi_{\mu} \psi_{\nu}}{2} .
\end{aligned}
$$

Expressions (6.51) to (6.54) look like those in [16] modulo this BRST exact term. This whole construction can be carried out on any symplectic manifold, where we can use Darboux coordinates. By the usual arguments, the BRST cohomology is concentrated in conformal weight zero, and the class of

$$
\mathcal{O}_{A}=A_{\mu_{1} \cdots \mu_{k}}(\gamma) \chi^{\mu_{1}} \cdots \chi^{\mu_{k}}
$$

is identified with the class $[A] \in H_{\mathrm{dR}}^{k}(M)$, the de Rham cohomology of $M$.

The B-twist corresponds to twisting the $N=2$ algebra $\left(J_{1}, G_{1}^{ \pm}, L_{1}\right)$. Redefining $L_{1} \rightarrow L_{1}+\frac{1}{2} \partial J_{1}$ and using holomorphic coordinates, the corresponding topological $N=2$ algebra $\left(L_{1}, J_{1}, Q_{1}, G_{1}\right)$ is given by

$$
\begin{aligned}
L_{1} & =\partial \gamma^{i} \beta_{i}+\partial \gamma^{\bar{i}} \beta_{\bar{i}}+\partial b_{i} c^{i}+\partial c^{\bar{i}} b_{\bar{i}}, \\
J_{1} & =b_{i} c^{i}-b_{\bar{i}} c^{\bar{i}} \\
Q_{1} & =G_{1}^{+}=\partial \gamma^{i} b_{i}+c^{\bar{i}} \beta_{\bar{i}}, \\
G_{1} & =G_{1}^{-}=\partial \gamma^{\bar{i}} b_{\bar{i}}+c^{i} \beta_{i} .
\end{aligned}
$$

With respect to the new Virasoro, the fields $b_{i}, b_{\bar{i}}, c^{i}, c^{\bar{i}}$ have conformal weights $0,1,1,0$, respectively. The two odd generators $Q_{1}$ and $G_{1}$ are of conformal weights 1 and 2. Thus the zero mode of $Q_{1}$ is a BRST operator. Using holomorphic coordinates we can glue all these formulas on a CalabiYau manifold. The BRST cohomology is concentrated in conformal weight 
zero, and the class of

$$
\mathcal{O}_{B}=B_{\bar{i}_{1} \ldots \bar{i}_{p}}^{j_{1} \ldots j_{q}}(\gamma) c^{\overline{i_{1}}} \ldots c^{\bar{i}_{p}} b_{j_{1}} \ldots b_{j_{q}}
$$

can be identified with the class $[B] \in H_{\bar{\partial}}^{p}\left(M, \wedge^{q} T_{M}^{1,0}\right)$.

\section{Summary}

In this work, we suggested to interpret $\Omega_{\hbar}^{c h}(M)$ as the prescription for a canonical quantization of the non-linear sigma model ${ }^{8}$. In [11] it has been shown that for a Calabi-Yau manifold $M$ with a fixed Ricci flat metric one can construct global sections of CDR which generate two commuting copies of $N=2$ superconformal algebra with central charge $\frac{3}{2} \operatorname{dim}_{\mathbb{R}} M$ each. The form of these global sections has been guessed in [11]. Here we show that these sections can be derived systematically from the sigma model action. Namely, they correspond to the Hamiltonian realization of $N=(2,2)$ superconformal symmetry for the sigma model with a Calabi-Yau manifold as target. We explain the matching of classical and quantum brackets, thus supporting the idea of the Hamiltonian interpretation of CDR. In the Hamiltonian formalism, the dynamics is introduced through the flow equations upon the choice of a Hamiltonian function. In fact, if we choose the correct Hamiltonian, CDR contains the classical dynamics of the non-linear sigma model. Amazingly, this is the same Hamiltonian which allows us to interpret the two copies of the $N=2$ algebra in CDR as left (chiral) and right (anti-chiral) sectors.

Let us make a side remark at this point. In the context of our interpretation, the ability to construct two commuting copies of $N=2$ (with the correct central charges!) attached to a Calabi-Yau manifold with a Ricci flat metric raises a puzzle about the multi-loop calculations for the supersymmetric sigma model. It is believed that the sigma model on a Calabi-Yau manifold is superconformal, but for a non-Ricci-flat metric. The reader may consult [18] for details. We hope to address this puzzle elsewhere.

The proposed interpretation of CDR also suggests some interesting mathematical ideas for vertex algebras. A vertex algebra can be understood as a quantum field theory in the Schrödinger picture. In particular, the $\beta \gamma b c$ system can be interpreted as a free boson and free fermion in the Schrödinger picture. Once the appropriate Hamiltonian is introduced, we can switch to

\footnotetext{
${ }^{8}$ Also see the related work by Malikov [17] on the relation of CDR and the Lagrangian approach.
} 
the Heisenberg picture by means of the flow equations. We hope that this idea may allow to incorporate both chiral and anti-chiral sectors in a single mathematical framework.

CDR should describe many systems which have $T^{*} \mathcal{L} M$ as a classical phase space. The dynamics would crucially depend on the concrete choice of Hamiltonian. Moreover, we can deal with the gauge systems on $T^{*} \mathcal{L} M$ (e.g., the Poisson sigma model, the non-linear model on $M / G$ when $M$ admits the action of a Lie group $G$, etc.). The appropriate BRST symmetries on CDR should be introduced to take care of the gauge symmetries, very much in the spirit of [3].

For example, in order to describe the system related to the large volume limit of the sigma model [7-9] we have to choose the following Hamiltonian:

$$
H=\mathrm{i} \int d \xi d \theta \xi\left(\partial \phi^{i} S_{i}-\partial \phi^{\bar{i}} S_{\bar{i}}\right),
$$

written in holomorphic coordinates. One can easily check that the corresponding flow equations admit holomorphic maps as solutions. We hope that one can derive the results from [7-9] in the present operatorial Hamiltonian framework. Indeed, it would be a good playground to check if we can describe the instanton correction within CDR with the Hamiltonian flow equations.

\section{Acknowledgments}

We thank Edward Frenkel, Andrei Losev, Joseph Minahan, and Konstantin Zarembo for illuminating discussions. R.H. and M.Z. thank KITP, Santa Barbara where part of this work was carried out. The research of R.H. and M.Z. was supported in part by DARPA under Grant No. HR0011-09-1-0015 and by the National Science Foundation under Grant No. PHY05-51164. The research of R.H. is supported by the Miller Institute for basic research in Science. The research of M.Z. is supported by VR-grant 621-2008-4273.

\section{Appendix A Quantum and classical brackets on Calabi-Yau}

On a Calabi-Yau manifold $M$, equipped with a complex structure $I$ and a symplectic structure $\omega$ such that $g=-\omega I$ is a Ricci-flat metric, we define the global sections of $\Omega_{\hbar}^{c h}(M)\left(\mathcal{P}, \mathcal{J}_{1}, \mathcal{J}_{2}, \mathcal{H}\right)$ given by (3.1), (3.2), (3.5), and (3.8), respectively. These sections form the following algebra with respect 
to the $\Lambda$-bracket $[11]$ :

$$
\begin{aligned}
{\left[\mathcal{P}_{\Lambda} \mathcal{P}\right] } & =\hbar(2 \partial+\chi D+3 \lambda) \mathcal{P}+\hbar^{2} \frac{c}{3} \lambda^{2} \chi, \\
{\left[\mathcal{P}_{\Lambda} \mathcal{J}_{1}\right] } & =\hbar(2 \partial+2 \lambda+\chi D) \mathcal{J}_{1}, \\
{\left[\mathcal{P}_{\Lambda} \mathcal{J}_{2}\right] } & =\hbar(2 \partial+2 \lambda+\chi D) \mathcal{J}_{2}, \\
{\left[\mathcal{J}_{1} \mathcal{J}_{1}\right] } & =-\hbar \mathcal{P}-\hbar^{2} \frac{c}{3} \lambda \chi, \\
{\left[\mathcal{J}_{2} \mathcal{J}_{2}\right] } & =-\hbar \mathcal{P}-\hbar^{2} \frac{c}{3} \lambda \chi, \\
{\left[\mathcal{J}_{1} \mathcal{J}_{2}\right] } & =-\hbar \mathcal{H}, \\
{\left[\mathcal{H}_{\Lambda} \mathcal{J}_{1}\right] } & =\hbar(2 \partial+\chi D+2 \lambda) \mathcal{J}_{2}, \\
{\left[\mathcal{H}_{\Lambda} \mathcal{J}_{2}\right] } & =\hbar(2 \partial+\chi D+2 \lambda) \mathcal{J}_{1}, \\
{\left[\mathcal{H}_{\Lambda} \mathcal{H}\right] } & =\hbar(2 \partial+\chi D+3 \lambda) \mathcal{P}+\hbar^{2} \frac{c}{3} \lambda^{2} \chi, \\
{\left[\mathcal{P}_{\Lambda} \mathcal{H}\right] } & =\hbar(2 \partial+\chi D+3 \lambda) \mathcal{H},
\end{aligned}
$$

where $c=3 \operatorname{dim}_{\mathbb{R}} M$. We introduce the following notations: $Z=(\xi, \theta)$ and $Z^{\prime}=\left(\xi^{\prime}, \theta^{\prime}\right), \partial^{\prime} \equiv \partial_{\xi^{\prime}}, D^{\prime}=D_{\xi^{\prime} \theta^{\prime}}, \delta\left(Z, Z^{\prime}\right)=\delta\left(\xi-\xi^{\prime}\right) \delta\left(\theta-\theta^{\prime}\right)$. Then the above algebra for the operators $\left(\mathcal{P}, \mathcal{J}_{1}, \mathcal{J}_{2}, \mathcal{H}\right)$ can be written equivalently in terms of commutators as

$$
\begin{aligned}
{\left[\mathcal{P}(Z), \mathcal{P}\left(Z^{\prime}\right)\right]=} & \hbar\left(2 \delta\left(Z, Z^{\prime}\right) \partial^{\prime}-D^{\prime} \delta\left(Z, Z^{\prime}\right) D^{\prime}+3 \partial^{\prime} \delta\left(Z, Z^{\prime}\right)\right) \mathcal{P}\left(Z^{\prime}\right) \\
& -\hbar^{2} \frac{c}{3} \partial^{\prime 2} D^{\prime} \delta\left(Z, Z^{\prime}\right), \\
{\left[\mathcal{P}(Z), \mathcal{J}_{i}\left(Z^{\prime}\right)\right]=} & \hbar\left(2 \delta\left(Z, Z^{\prime}\right) \partial^{\prime}+2 \partial^{\prime} \delta\left(Z, Z^{\prime}\right)-D^{\prime} \delta\left(Z, Z^{\prime}\right) D^{\prime}\right) \mathcal{J}_{i}\left(Z^{\prime}\right), \\
{\left[\mathcal{J}_{i}(Z), \mathcal{J}_{i}\left(Z^{\prime}\right)\right]=} & -\hbar \delta\left(Z, Z^{\prime}\right) \mathcal{P}\left(Z^{\prime}\right)+\hbar^{2} \frac{c}{3} \partial^{\prime} D^{\prime} \delta\left(Z, Z^{\prime}\right), \\
{\left[\mathcal{J}_{1}(Z), \mathcal{J}_{2}\left(Z^{\prime}\right)\right]=} & -\hbar \delta\left(Z, Z^{\prime}\right) \mathcal{H}\left(Z^{\prime}\right), \\
{\left[\mathcal{H}(Z), \mathcal{J}_{1}\left(Z^{\prime}\right)\right]=} & \hbar\left(2 \delta\left(Z, Z^{\prime}\right) \partial^{\prime}-D^{\prime} \delta\left(Z, Z^{\prime}\right) D^{\prime}+2 \partial^{\prime} \delta\left(Z, Z^{\prime}\right)\right) \mathcal{J}_{2}\left(Z^{\prime}\right), \\
{\left[\mathcal{H}(Z), \mathcal{J}_{2}\left(Z^{\prime}\right)\right]=} & \hbar\left(2 \delta\left(Z, Z^{\prime}\right) \partial^{\prime}-D^{\prime} \delta\left(Z, Z^{\prime}\right) D^{\prime}+2 \partial^{\prime} \delta\left(Z, Z^{\prime}\right)\right) \mathcal{J}_{1}\left(Z^{\prime}\right), \\
{\left[\mathcal{H}(Z), \mathcal{H}\left(Z^{\prime}\right)\right]=} & \hbar\left(2 \delta\left(Z, Z^{\prime}\right) \partial^{\prime}-D^{\prime} \delta\left(Z, Z^{\prime}\right) D^{\prime}+3 \partial^{\prime} \delta\left(Z, Z^{\prime}\right)\right) \mathcal{P}\left(Z^{\prime}\right) \\
& -\hbar^{2} \frac{c}{3} \partial^{\prime 2} D^{\prime} \delta\left(Z, Z^{\prime}\right), \\
{\left[\mathcal{P}(Z), \mathcal{H}\left(Z^{\prime}\right)\right]=} & \hbar\left(2 \delta\left(Z, Z^{\prime}\right) \partial^{\prime}-D^{\prime} \delta\left(Z, Z^{\prime}\right) D^{\prime}+3 \partial^{\prime} \delta\left(Z, Z^{\prime}\right)\right) \mathcal{H}\left(Z^{\prime}\right) .
\end{aligned}
$$


If we take the classical limit $\hbar \rightarrow 0$ and make the replacement [,] $\rightarrow \hbar\{$,$\} ,$ then we can view this algebra as a super-Poisson subalgebra of the Poisson algebra of functionals on $T^{*} \mathcal{L} M$, the cotangent bundle of the superloop space. Using the following conventions for the local functional $F$ :

$$
\delta F=\int d \xi d \theta\left(\frac{F \overleftarrow{\delta}}{\delta S_{\mu}} \delta S^{\mu}+\frac{F \overleftarrow{\delta}}{\delta \Phi^{\mu}} \delta \Phi^{\mu}\right)=\int d \xi d \theta\left(\delta S_{\mu} \frac{\vec{\delta} F}{\delta S_{\mu}}+\delta \Phi^{\mu} \frac{\vec{\delta} F}{\delta \Phi^{\mu}}\right)
$$

we find the corresponding Poisson bracket between the local functional $F$ and $G$

$$
\{F, G\}=\int d \xi d \theta\left(\frac{F \overleftarrow{\delta}}{\delta S_{\mu}} \frac{\vec{\delta} G}{\delta \Phi^{\mu}}-\frac{F \overleftarrow{\delta}}{\delta \Phi^{\mu}} \frac{\vec{\delta} G}{\delta S_{\mu}}\right)
$$

This bracket is super-Poisson. In particular, we are interested in the local expressions $F(\xi, \theta)$,

$$
F(\xi, \theta)=F(\phi, S, D \phi, D S, \ldots),
$$

which are constructed from the basic fields and a finite number of derivatives of those fields. This local expression $F(\xi, \theta)$ can be interpreted as a functional evaluated at the point $(\xi, \theta)$. These local expressions can be multiplied and thus they form a super-Poisson subalgebra of the super-Poisson algebra of all functionals on $T^{*} \mathcal{L} M$. This super-Poisson subalgebra of local expressions is the most interesting from the physics point of view. If we approach this subalgebra formally we end up with the notion of Poisson vertex algebra. The Poisson brackets between the classical generators defined in (4.16) to (4.19) are given by the following expressions:

$$
\begin{aligned}
&\left\{\mathcal{P}(Z), \mathcal{P}\left(Z^{\prime}\right)\right\}=\left(2 \delta\left(Z, Z^{\prime}\right) \partial^{\prime}-D^{\prime} \delta\left(Z, Z^{\prime}\right) D^{\prime}+3 \partial^{\prime} \delta\left(Z, Z^{\prime}\right)\right) \mathcal{P}\left(Z^{\prime}\right) \\
&\left\{\mathcal{P}(Z), \mathcal{J}_{i}\left(Z^{\prime}\right)\right\}=\left(2 \delta\left(Z, Z^{\prime}\right) \partial^{\prime}+2 \partial^{\prime} \delta\left(Z, Z^{\prime}\right)-D^{\prime} \delta\left(Z, Z^{\prime}\right) D^{\prime}\right) \mathcal{J}_{i}\left(Z^{\prime}\right) \\
&\left\{\mathcal{J}_{i}(Z), \mathcal{J}_{i}\left(Z^{\prime}\right)\right\}=-\delta\left(Z, Z^{\prime}\right) \mathcal{P}\left(Z^{\prime}\right), \\
&\left\{\mathcal{J}_{1}(Z), \mathcal{J}_{2}\left(Z^{\prime}\right)\right\}=-\delta\left(Z, Z^{\prime}\right) \mathcal{H}\left(Z^{\prime}\right), \\
&\left\{\mathcal{H}(Z), \mathcal{J}_{1}\left(Z^{\prime}\right)\right\}=\left(2 \delta\left(Z, Z^{\prime}\right) \partial^{\prime}-D^{\prime} \delta\left(Z, Z^{\prime}\right) D^{\prime}+2 \partial^{\prime} \delta\left(Z, Z^{\prime}\right)\right) \mathcal{J}_{2}\left(Z^{\prime}\right) \\
&\left\{\mathcal{H}(Z), \mathcal{J}_{2}\left(Z^{\prime}\right)\right\}=\left(2 \delta\left(Z, Z^{\prime}\right) \partial^{\prime}-D^{\prime} \delta\left(Z, Z^{\prime}\right) D^{\prime}+2 \partial^{\prime} \delta\left(Z, Z^{\prime}\right)\right) \mathcal{J}_{1}\left(Z^{\prime}\right)
\end{aligned}
$$




$$
\begin{aligned}
& \left\{\mathcal{H}(Z), \mathcal{H}\left(Z^{\prime}\right)\right\}=\left(2 \delta\left(Z, Z^{\prime}\right) \partial^{\prime}-D^{\prime} \delta\left(Z, Z^{\prime}\right) D^{\prime}+3 \partial^{\prime} \delta\left(Z, Z^{\prime}\right)\right) \mathcal{P}\left(Z^{\prime}\right), \\
& \left\{\mathcal{P}(Z), \mathcal{H}\left(Z^{\prime}\right)\right\}=\left(2 \delta\left(Z, Z^{\prime}\right) \partial^{\prime}-D^{\prime} \delta\left(Z, Z^{\prime}\right) D^{\prime}+3 \partial^{\prime} \delta\left(Z, Z^{\prime}\right)\right) \mathcal{H}\left(Z^{\prime}\right) .
\end{aligned}
$$

\section{References}

[1] L. Baulieu, A. S. Losev and N. A. Nekrasov, Target space symmetries in topological theories. I, JHEP 0202 (2002), 021; arXiv:hep-th/0106042.

[2] D. Ben-Zvi, R. Heluani and M. Szczesny, Supersymmetry of the chiral de Rham complex, Compo. Math., 144(2) (2008), 503-521; arXiv: math.QA/0601532.

[3] L. A. Borisov, Vertex algebras and mirror symmetry, Commun. Math. Phys. 215 (2001), 517; arXiv:math/9809094.

[4] A. Bredthauer, U. Lindström, J. Persson and M. Zabzine, Generalized Kähler geometry from supersymmetric sigma models, Lett. Math. Phys. 77 (2006), 291; arXiv:hep-th/0603130.

[5] A. De Sole and V. Kac, Finite vs. Affine $W$-algebras, Jpn. J. Math. 1 (1) (2006), 137-261.

[6] E. Frenkel and D. Ben-Zvi, Vertex algebras and algebraic curves, Mathematical surveys and monographs v 88, AMS, Providence, RI, 2001.

[7] E. Frenkel and A. Losev, Mirror symmetry in two steps: A-I-B, Commun. Math. Phys. 269 (2007), 39; arXiv: hep-th/0505131.

[8] E. Frenkel, A. Losev and N. Nekrasov, Instantons beyond topological theory. I; arXiv:hep-th/0610149.

[9] E. Frenkel, A. Losev and N. Nekrasov, Instantons beyond topological theory II; arXiv:0803.3302 [hep-th].

[10] R. Heluani and V. Kac, Super symmetric vertex algebras, Commun. Math. Phys. 271 (2007), 103; arXiv:math/0603633.

[11] R. Heluani, Supersymmetry of the chiral de Rham complex II: commuting sectors, Int. Math. Res. Not. 2009 (2009), 953-987; arXiv:0806.1021.

[12] R. Heluani and M. Zabzine, Generalized Calabi-Yau manifolds and the chiral de Rham complex; arXiv:0812.4855 [math.QA].

[13] V. Kac, Vertex algebras for beginners, (University Lectures Series, 10). AMS, Providence, USA, 1996, 141p.

[14] A. Kapustin, Chiral de Rham complex and the half-twisted sigma-model; arXiv:hep-th/0504074. 
[15] B. Lian and A. Linshaw, Chiral equivariant cohomology I, Adv. Math. 209 (2007), 99; arXiv:math/0501084.

[16] F. Malikov, V. Schechtman and A. Vaintrob, Chiral de Rham complex, Commun. Math. Phys. 204 (1999), 439; arXiv:math/9803041.

[17] F. Malikov, Lagrangian approach to sheaves of vertex algebras, Commun. Math. Phys. 278 (2008), 487; arXiv:math/0604093.

[18] D. Nemeschansky and A. Sen, Conformal invariance of supersymmetric $\sigma$-models on Calabi-Yau manifolds, Phys. Lett. B 178 (1986), 365.

[19] M. C. Tan, Two-dimensional twisted sigma models and the theory of chiral differential operators, Adv. Theor. Math. Phys. 10 (2006), 759; arXiv: hep-th/0604179.

[20] M. C. Tan, Two-dimensional twisted sigma models, the mirror chiral de rham complex, and twisted generalised mirror symmetry, JHEP 0707 (2007), 013; arXiv:0705.0790 [hep-th].

[21] E. Witten, Two-dimensional models with (0,2) supersymmetry: perturbative aspects, Adv. Theor. Math. Phys. 11 (1) (2007), 1-63. arXiv:hep-th/0504078.

[22] M. Zabzine, Hamiltonian perspective on generalized complex structure, Commun. Math. Phys. 263 (2006), 711; arXiv:hep-th/0502137.

[23] M. Zabzine, Lectures on generalized complex geometry and supersymmetry, Arch. Math. (Suppl.) 42 (2006), 119-146; arXiv:hep-th/0605148. 